\title{
Estructura y dinámica del manglar del delta del río Ranchería, Caribe colombiano
}

\author{
Luisa Fernanda Lema Vélez ${ }^{1} \&$ Jaime Polanía ${ }^{2}$ \\ Universidad Nacional de Colombia Sede Medellín; luisalema@aya.yale.edu \\ Universidad Nacional de Colombia Sede Medellín, Calle 59 A No, 62-00 Autopista Norte, Medellín, Colombia. Fax: \\ 57-4-4309579; jhpolanv@unalmed.edu.co
}

Recibido 20-XI-2005. Corregido 26-VI-2006. Aceptado 15-XII-2006.

\begin{abstract}
Structure and dynamics of the mangrove forest in the Ranchería river delta, Colombian Caribbean. We registered seedling survival and biomass increase for Rhizophora mangle L., Avicennia germinans L. and Laguncularia racemosa (L.) Gaertn. f., main mangrove species in the Rancheria River delta, Colombia. Only seedlings of $R$. mangle were found to survive. We also measured maximum rate of litterfall. We estimated annual litterfall through interpolation within an exponential regression performed with maximum and annual litterfall data published in other sources; the value of annual litterfall for the area was estimated to be $12.9 \mathrm{mgha}^{-1} \mathrm{y}^{-1}$. We found a $7.4 \mathrm{mgha}^{-1} \mathrm{y}^{-1}$ increase in biomass. Litterfall constitutes the larger fraction of the $20.2 \mathrm{mgha}^{-1} \mathrm{y}^{-1}$ productivity of this mangrove. We believe this is a very high value for a forest under unfavorable natural and human conditions, such as high seasonality and continuous use of the forest to feed goats and sheep. We consider that the high productivity is a response to both natural and anthropogenic stress. Rev. Biol. Trop. 55 (1): 11-21. Epub 2007 March. 31.
\end{abstract}

Key words: mangrove, litterfall, growth, survival, productivity.

Los manglares son asociaciones de especies que por su tolerancia a la salinidad y a los sustratos inestables pueden establecerse en zonas costeras y ribereñas con influencia mareal. La productividad de los manglares está entre las mayores de los ecosistemas costeros (Lacerda et al. 2001). Son ecosistemas estratégicos y vitales para las comunidades adyacentes porque a través de las corrientes de agua exportan gran cantidad de material orgánico. La calidad y la cantidad de los sedimentos y la materia orgánica exportada dependen del tipo de bosque de manglar, de su productividad y de factores limitantes físicos y biológicos. La abundante hojarasca que producen (Gattuso et al. 1998) es esencial para la regeneración de los nutrimentos en suelos, su exportación a cadenas tróficas costeras, en especial microbianas (Wafar et al. 1997, Machiwa y Hallberg
2002), y la dinámica del carbono en sistemas litorales (Wafar et al. 1997, Gattuso et al. 1998, Alongi et al. 2001).

La productividad de los manglares, cuantificada a partir del aumento de biomasa aérea o de la caída de hojarasca, ha sido estudiada profusamente y las metodologías de campo para medirla están más o menos estandarizadas. Saenger y Snedaker (1993) recopilaron trabajos en todo el mundo que medían la productividad en manglares. Existen además estudios nacionales más recientes en Australia (Bunt 1995); Brasil (Mehlig 2001); Ecuador (Twilley et al. 1997); Florida, USA (Twilley et al. 1986, Chen y Twilley 1999, Ross et al. 2001); Guyana Francesa (Fromard et al. 1998, Betoulle et al. 2001); Malasia (Jin-Eong et al. 1995, Wafar et al. 1997, Tanouchi et al. 2000); México (Day et al. 1996, Barreiro-Güemes 
1999) y la Ciénaga Grande de Santa Marta, Colombia (Zamorano 1983).

Además de ser el principal patrimonio paisajístico en la urbe, el manglar de Riohacha (Caribe colombiano) es un caso interesante desde el punto de vista ecológico, ya que se desarrolla en condiciones de mínima precipitación, períodos áridos prolongados (estación seca) y considerables de inundación (estación lluviosa), elevada insolación y alta presión antrópica. Un proyecto marco permitió caracterizar la estructura del bosque, estudiar la edafología, la hidrología, la fenología, la dispersión de propágulos, el establecimiento, la supervivencia y la disponibilidad de plántulas, el crecimiento de estados inmaduros, la sucesión vegetal en el Holoceno y determinar relaciones alométricas. Con este estudio se pretende evaluar la tasa de supervivencia de la regeneración natural y generar información sobre productividad de un manglar en condiciones extremas de sitio y clima.

\section{MATERIALES Y MÉTODOS}

Área de estudio: el delta del río Ranchería se ubica a $11^{\circ} 33^{\prime} \mathrm{N}, 72^{\circ} 53^{\prime}-72^{\circ} 54^{\prime} \mathrm{W}$ (Fig. 1). La planicie deltáica se encuentra en una transición entre las zonas de vida bs-ST y me-ST, según la clasificación de Holdridge (1978). La precipitación media anual es de $554 \mathrm{~mm}$, con distribución bimodal y un pico máximo entre septiembre y octubre. La temperatura media anual es de $27^{\circ} \mathrm{C}$, con el período más cálido entre junio y agosto. La humedad relativa media anual es $73 \%$ y la evaporación media de $2293 \mathrm{~mm} \mathrm{a}^{-1}$ (Anónimo 1994b). Los vientos son fuertes una buena parte del año y registran una velocidad promedio en superficie de 30 a $40 \mathrm{~cm} \mathrm{~s}^{-1}$ en dirección NE (Cuignon 1987).

La formación deltáica está compuesta por tres brazos principales, denominados Riíto (en adelante RT), Santa Rita y Calancala, y varias lagunas costeras. Se denomina Valle de los Cangrejos (VC) a la ribera izquierda del brazo Calancala, en la cual se encuentra un área inundada periódicamente por desbordamiento en la estación lluviosa. En la estación seca suele detenerse el flujo del agua y se suspende temporalmente la conexión del sistema de cauces con el mar. La salinidad intersticial y el nivel del agua fluctúan ampliamente durante el año (Fig. 2).

El manglar de RT es de tipo ribereño, dominado por Laguncularia racemosa, cuyo diámetro normal promedio es $7.8 \mathrm{~cm}$ y el máximo es $43.7 \mathrm{~cm}$. El manglar de VC es de tipo cuenca, dominado por Avicennia germinans; los árboles son de porte bajo, con diámetro normal promedio de $5.1 \mathrm{~cm}$ y máximo de $48.7 \mathrm{~cm}$. En VC se encuentran exclusivamente especies de manglar, mientras que en RT se presentan otras especies halófitas en muy baja frecuencia (Vásquez 2000; Cuadros 1 y 2). RT recibe descargas de aguas servidas de la ciudad de Riohacha, que producen desalinización localizada (Martínez-Ramos y Ruiz-Ochoa 2001), y promueven la presencia de L racemosa y Rhizophora mangle (Lema et al. 2002). Algunas de las lagunas originales del sistema deltáico han sido desecadas para extensión del área urbana. El manglar es también un área de tránsito para las comunidades vecinas de la

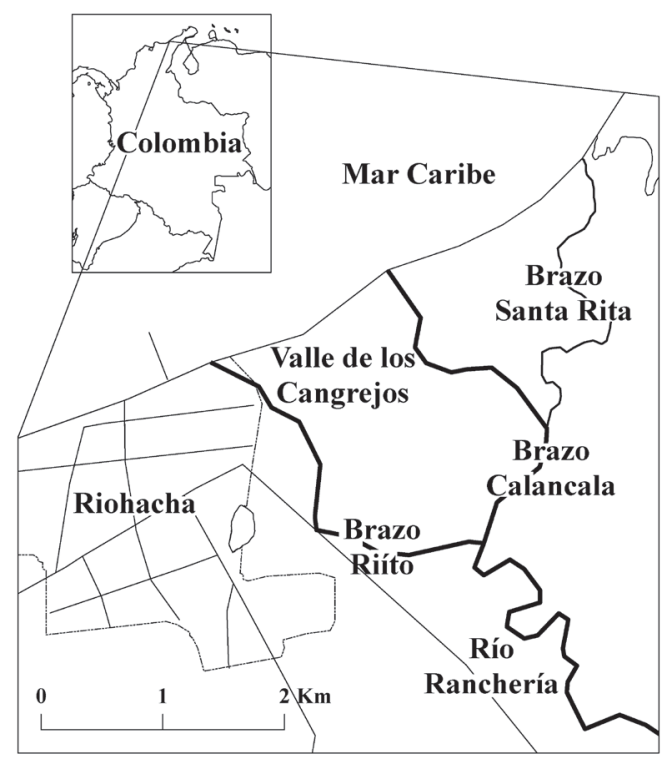

Fig. 1. Localización del delta del río Ranchería.

Fig. 1. Río Ranchería delta. 


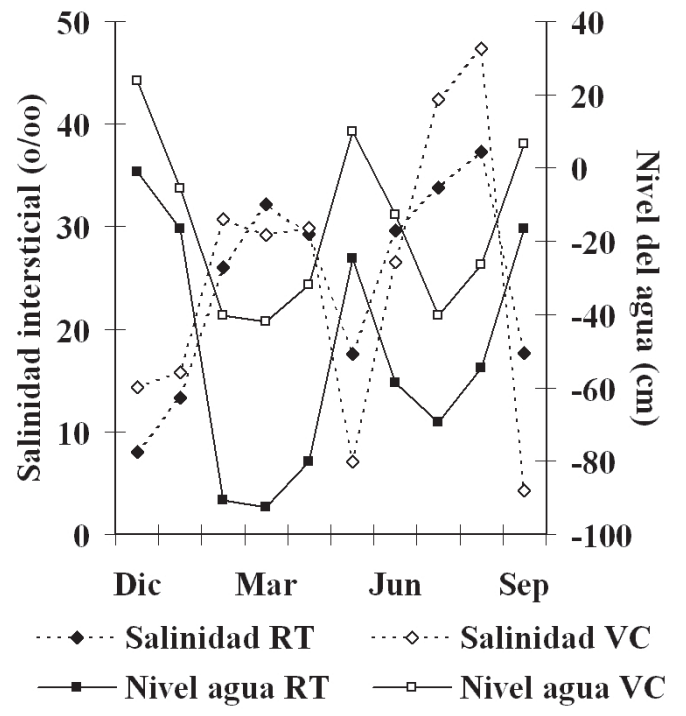

Fig. 2. Salinidad en agua intersticial y nivel del agua en el delta del río Ranchería. Datos de Maza y Zárate (2001), y Barros et al. (2002); promedio 2000-2001.

Fig. 2. Interstitial salinity and water level at the Rancheria river delta. Data from Maza and Zárate (2001), and Barros et al. (2002); average for 2000-2001. etnia Wayuú, quienes lo usan para leña y como forraje para ganado caprino y ovino.

Para este estudio se establecieron siete corredores paralelos a la línea de costa, cinco en RT y dos en $\mathrm{VC}$, todos ellos de $40 \mathrm{~m}$ de ancho y longitud variable según la extensión del bosque. En total se establecieron 58 unidades cuadradas de $20 \mathrm{~m}$ de lado. En mayo y junio de 2000 se midieron el diámetro, altura total, altura a la primera rama y altura de raíz, entre otros parámetros de estructura de bosque, en un $65 \%$ de las unidades (Vásquez 2000). Estos parámetros fueron remedidos en mayo de 2002 sobre individuos marcados (15 $R$. mangle, 14 A. germinans y 15 L. racemosa), para determinar el crecimiento del bosque. En corredores de $10 \mathrm{~m}$ de ancho, adyacentes a los anteriores, se rotularon con placas de aluminio 100 plántulas de L. racemosa, 100 de $A$. germinans y 50 de $R$. mangle en RT, y 100 de $A$. germinans y 50 de $R$. mangle en VC (Díaz-Fuenmayor 2001). La supervivencia de las plantas se monitoreó cada diez días entre

CUADRO 1

Índice de valor de importancia (IVI) para las especies encontradas en el manglar del delta del rio Ranchería

TABLE 1

Importance value index (IVI) for mangrove species in the Rancheria River delta

\begin{tabular}{ccccccccccccc} 
& \multicolumn{3}{c}{ Brazo Riíto } & \multicolumn{4}{c}{ Valle de los Cangrejos } & \multicolumn{3}{c}{ Promedio para el delta } \\
& Dom & Abu & Fre & IVI & Dom & Abu & Fre & IVI & Dom & Abu & Fre & IVI \\
$\mathrm{Ag}$ & 35.7 & 41.9 & 26.2 & 103.8 & 67.7 & 39.2 & 57.1 & 164.1 & 46.5 & 40.4 & 38.6 & 125.4 \\
$\mathrm{Lr}$ & 51.7 & 46.9 & 33.3 & 131.9 & $25, .6$ & 57.5 & 25.0 & 108.1 & 42.9 & 52.9 & 30.0 & 125.8 \\
$\mathrm{Rm}$ & 7.7 & 4.6 & 16.7 & 29.0 & 6.7 & 3.1 & 14.3 & 24.1 & 7.4 & 3.8 & 15.7 & 26.8 \\
$\mathrm{Ce}$ & 3.9 & 2.9 & 9.5 & 16.4 & 0.0 & 0.2 & 3.6 & 3.8 & 2.6 & 1.4 & 7.1 & 11.1 \\
$\mathrm{Rb}$ & 0.0 & 0.5 & 7.1 & 7.7 & 0.0 & 0.0 & 0.0 & 0.0 & 0.0 & 0.2 & 4.3 & 4.5 \\
$\mathrm{Pj}$ & 0.6 & 0.9 & 4.8 & 6.3 & 0.0 & 0.0 & 0.0 & 0.0 & 0.4 & 0.4 & 2.9 & 3.6 \\
$\mathrm{Co}$ & 0.4 & 2.2 & 2.4 & 5.1 & 0.0 & 0.0 & 0.0 & 0.0 & 0.3 & 1.0 & 1.4 & 2.7
\end{tabular}

Dom: dominancia, Abu: abundancia, Fre: frecuencia; Ag: A. germinans; Lr: L. racemosa; Rm: R. mangle; Ce: Conocarpus erecta; Pj: Prosopis juliflora; Co: Capparis odoratissima; Rb: Rhabdadenia biflora (modificado de Vásquez 2000).

Dom: dominance, Abu: abundance, Fre: frequency; Ag: A. germinans; Lr: L. racemosa; Rm: R. mangle; Ce: Conocarpus erecta; Pj: Prosopis juliflora; Co: Capparis odoratissima; Rb: Rhabdadenia biflora (modified from Vásquez 2000). 
CUADRO 2

Características estructurales de los bosques de manglar en el delta del río Ranchería

TABLE 2

Structural characteristics of mangrove forests at the Rancheria River delta

\begin{tabular}{lccccc}
\multicolumn{1}{c}{ Sitio } & $\mathrm{N}\left(\right.$ ind ha $\left.^{-1}\right)$ & $\mathrm{H}(\mathrm{m})$ & $\mathrm{D}(\mathrm{cm})$ & $\mathrm{G}\left(\mathrm{m}^{2} \mathrm{ha}^{-1}\right)$ & $\begin{array}{c}\mathrm{N}^{\circ} \text { de especies } \\
\text { arbóreas }\end{array}$ \\
Brazo Ríto & 1449 & 6.1 & 7.8 & 13.3 & 6 \\
Valle de los Cangrejos & 1557 & 4.4 & 5.1 & 5.5 & 4 \\
Promedio para el delta & 1509 & 4.9 & 6.5 & 9.0 & 6
\end{tabular}

N: número de árboles por hectárea, H: altura promedio, D: diámetro normal promedio, G: área basal por hectárea (tomado de Vásquez 2000).

$\mathrm{N}$ : number of trees per hectare, H: medium height, D: mean normal diameter, G: basal area per hectare (source: Vásquez 2000).

diciembre de 2000 y abril de 2001 y luego en mayo de 2002. Se generaron ecuaciones de biomasa total y por compartimentos a partir datos de 22 A. geminans y 20 L. racemosa extraídos del bosque para este fin (Correa 2002). Con estas ecuaciones y la de Fromard et al. (1998) para R. mangle (Cuadro 3), se calculó la biomasa total del bosque y la tasa de renovación foliar, expresada como el número de días que tarda un árbol en renovar su copa.

Para cuantificar la caída de hojarasca se demarcó el suelo de RT en cinco cuadrantes de $1 \mathrm{~m}^{2}$. En cada uno de ellos se recolectó todo el material vegetal acumulado cada segundo día durante dos semanas en mayo de 2002. El material fue secado en horno a $80^{\circ} \mathrm{C}$ hasta peso constante. Mayo representa el final de la estación seca mayor, inmediatamente después de un pico en la salinidad intersticial (Fig. 2), período al que Twilley et al. (1986) hacen referencia como de máxima caída de hojarasca. Se asumió esta medición de hojarasca como pico máximo y se estimó el valor anual interpolándolo en una regresión de valor diario máximo vs. valor anual de hojarasca, ajustada con registros de Twilley et al. (1986, 1997), Barreiro-Güemes (1999) y Mehling (2001).

CUADRO 3

Ecuaciones para estimación de biomasa a partir de diámetro

TABLE 3

Equations used for estimating biomass

\begin{tabular}{lccccc}
\multicolumn{1}{c}{ Especie } & Relación & $\mathrm{R}^{2}$ & Valor $\mathrm{p}$ & $\mathrm{n}$ & Fuente \\
L. racemosa & $\mathrm{BT}=0.147 * \mathrm{DAP}{ }^{2.4}$ & 98.2 & 0.0000 & 19 & Correa 2002 \\
A. germinans & $\mathrm{BT}=0.227 * \mathrm{DAP}^{2.3}$ & 97.3 & 0.0000 & 21 & Correa 2002 \\
R. mangle & $\mathrm{BT}=0.128 * \mathrm{DAP}^{2.6}$ & 92.0 & $<0.0001$ & 9 & Fromard et al. 1998
\end{tabular}

Las ecuaciones de Correa (2002) fueron generadas con datos del sitio de estudio y la de Fromard et al. con datos de Guyana Francesa. El diámetro a la altura del pecho (DAP) está dado en centímetros y la biomasa total (BT) en kilogramos.

The equations taken from Correa (2002) where generated with data from the study site, while that from Fromard et al. (1998) comes from data registered in French Guyana. Diameter at breast height (DAP) in centimeters and total biomass (BT) in kilograms. 
La productividad del manglar se estimó a partir del aumento en biomasa por año y la producción anual de hojarasca estimada como se describe arriba. El aporte a la productividad por reclutamiento de plántulas no fue considerado porque las supervivencias registradas fueron extremadamente bajas.

\section{RESULTADOS}

El incremento en diámetro promedio registrado para cada especie se muestra en el Cuadro 4 y las ecuaciones de crecimiento diamétrico en la Figura 3. Avicennia germinans fue la especie que mostró mayor crecimiento relativo (5.7\% de incremento diamétrico anual). El Cuadro 5 muestra la producción por especie en los dos
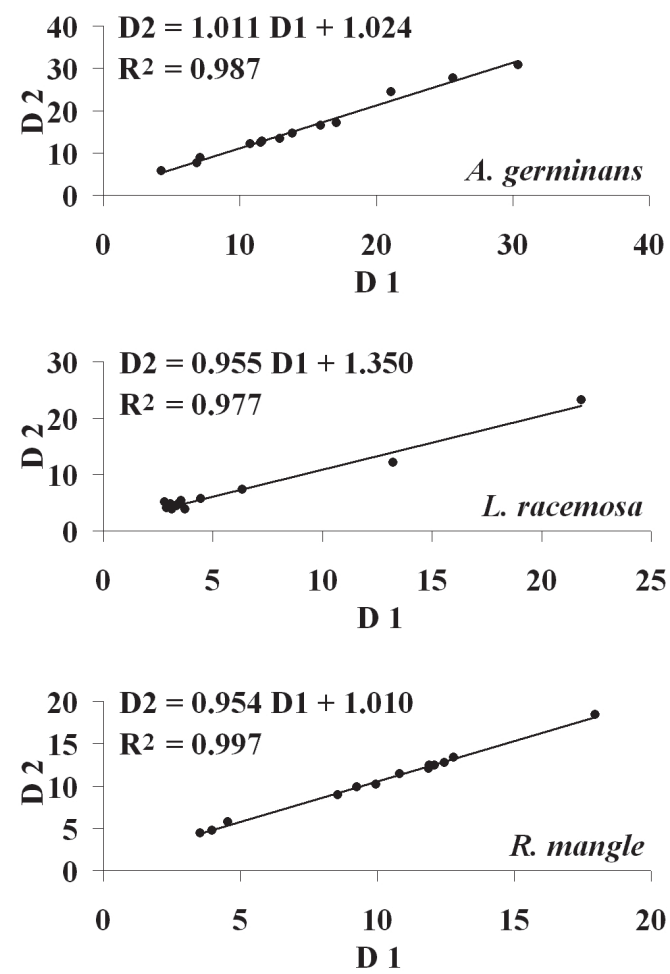

Fig. 3. Ecuaciones de crecimiento diamétrico para las especies más importantes del manglar del Río Ranchería. D1: diámetro inicial; D2: diámetro luego de dos años. Todas las ecuaciones son de tipo lineal.

Fig. 3. Diametric growth equations for the most important species in the Rancheria river mangrove forest. D1: initial diameter; D2: diameter after two years. All equations are linear. bosques estudiados; en el bosque de rivera en RT el valor duplica al del bosque de cuenca de VC (70.9 vs. 26.8 mgha $\left.^{-1}\right)$. Entre tanto, en el nivel de especie, L. racemosa aporta mayor biomasa en RT (52.4\%), mientras que $A$. germinans contribuye con $72.3 \%$ en $\mathrm{VC}$; en promedio, A. germinans participa más en la biomasa del delta $(49.5 \%)$ gracias a su alta representación en el VC. En total, el bosque en pie aporta $7.4 \mathrm{mgha}^{-1}$ de biomasa al año al ecosistema. La biomasa foliar se calculó como $5953.1 \mathrm{kgha}^{-1}$, lo cual indica una tasa de renovación foliar de 169 días en promedio para las tres especies de manglar.

La Figura 4 muestra el porcentaje de supervivencia registrado. Un año y cinco meses después de iniciado el monitoreo sólo sobrevivió el $28 \%$ de las plántulas de $R$. mangle en $\mathrm{RT}$, mientras que en $\mathrm{VC}$ no se registró supervivencia para ninguna de las especies.

Se midieron $5.9 \mathrm{~g} \mathrm{~m}^{-2} \mathrm{~d}^{-1}$ de hojarasca; asumiendo este valor como máximo en el año (pico de la salinidad intersticial; sensu Lugo y Snedaker 1974, Twilley et al. 1986), la productividad del área de estudio de estimó en 12.9 mgha $^{-1} \mathrm{a}^{-1}$, con probabilidad de $95 \%$ entre 10.2 y 16.1 mgha $^{-1} \mathrm{a}^{-1}$ (Fig. 5).

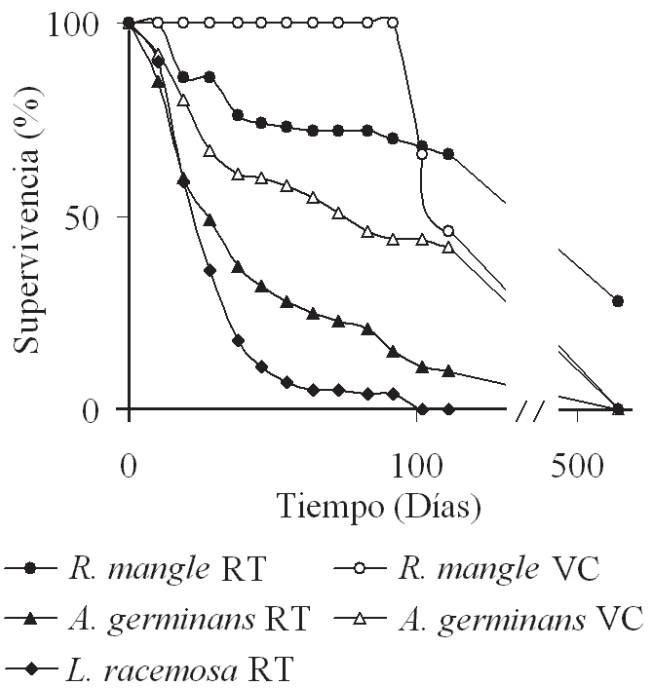

Fig. 4. Supervivencia de las plántulas de las principales especies de manglar en el delta del río Ranchería.

Fig. 4. Seedling survival for the main mangrove species at Rancheria River delta. 
CUADRO 4

Crecimiento diamétrico estimado para el área del brazo Riito

TABLE 4

Diametric growth estimated for the Riito area

\begin{tabular}{lcccc}
\multicolumn{1}{c}{ Especie } & $\mathrm{Dq}_{1}(\mathrm{~cm})$ & $\mathrm{Dq}_{2}(\mathrm{~cm})$ & $\Delta \mathrm{Dq}\left(\mathrm{cm} \mathrm{a}^{-1}\right)$ & $\Delta \mathrm{Dq}(\%)$ \\
A. germinans & 10.0 & 11.1 & 0.6 & 5.7 \\
L. racemosa & 11.4 & 12.2 & 0.4 & 3.7 \\
R. mangle & 14.1 & 14.4 & 0.2 & 1.3 \\
Promedio & 11.8 & 12.6 & 0.4 & 3.6
\end{tabular}

Dq: diámetro promedio cuadrático.

Dq: quadratic diameter.

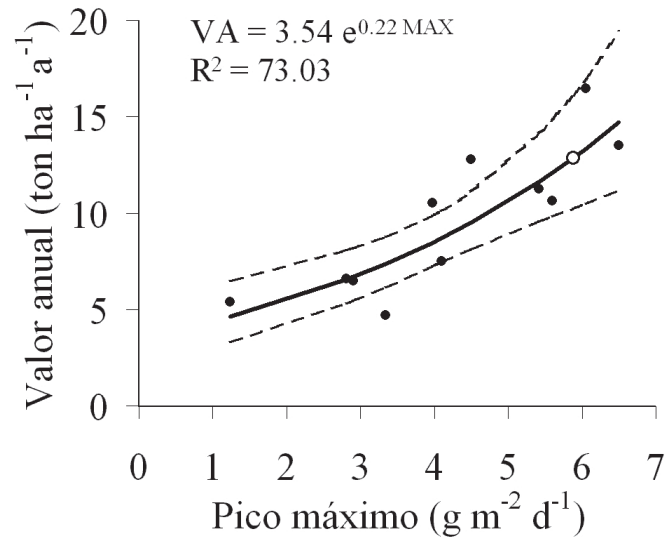

Fig. 5. Relación entre pico máximo y valor anual total de producción de hojarasca. VA: valor anual; MAX: valor mensual máximo. El punto vacío muestra el valor proyectado para el área de estudio. Límites de confianza al $95 \%$. Regresión a partir de datos en Twilley et al. 1986, 1997, Barreiro-Güemes 1999 y Mehling 2001.

Fig. 5. Relation between maximum and annual litterfall rates. VA: annual litterfall; MAX: maximum monthly litterfall. The white circle indicates the projected value for the study site. $95 \%$ confidence limits. Regression done with data from Twilley et al. 1986, 1997, Barreiro-Güemes 1999 y Mehling 2001.

El delta del río Ranchería presentó una productividad total de $20.2 \mathrm{mgha}^{-1} \mathrm{a}^{-1}$ (ámbito 17.6 y $23.5 \mathrm{mgha}^{-1} \mathrm{a}^{-1}$, con $95 \%$ de confianza), donde la caída de hojarasca representa $63.6 \%$. La regeneración no fue considerada significativa para la productividad del bosque, dada la baja supervivencia encontrada.

\section{DISCUSIÓN}

Las observaciones de supervivencia acá presentadas (Fig. 4) son comparables con las encontradas en otras áreas del Caribe Colombiano, como el antiguo delta del río Sinú y la Ciénaga Grande de Santa Marta, donde $R$. mangle mostró mayor potencial de regeneración frente a $A$. germinans y $L$. racemosa (Anónimo 1994a, Elster 1998). Ensayos de campo en otros manglares del Caribe también han mostrado que $R$. mangle tiene una mayor supervivencia y vida media, comparada con las demás especies de manglar (Rabinowitz 1978a, b, Ellison y Farnsworth 1993, McKee 1995). Finalmente, las altas tasas de mortalidad de plántulas de manglar como resultado de períodos de intensa sequía han sido informadas previamente en Costa Rica (Jiménez 1990) y Colombia (Sánchez-Páez et al. 1998).

Atribuimos la elevada mortalidad de plántulas a la baja disponibilidad de agua, alta irradiación, y a la depredación por ganado ovino y caprino. Nuestros resultados son consistentes en cuanto a la mayor capacidad de supervivencia y la longevidad de las plántulas de $R$. mangle con respecto a las otras especies en condiciones de relativa aridez. El tamaño y estructura inicial de los propágalos de $R$. mangle representan una ventaja ante la presencia de períodos extensos de alta inundación. Nuestros resultados confirman pues la alta sensibilidad 
CUADRO 5

Biomasa estimada para los bosques del delta del río Ranchería

TABLE 5

Estimated biomass for the Rancheria River delta forests

Densidad (ind/ha) Dq1 (cm) $\quad \begin{aligned} & \text { BT 2000 } \\ & \left(\mathrm{kg} \mathrm{ha}^{-1}\right)\end{aligned} \quad$ Dq2 (cm) $\quad \begin{aligned} & \text { BT 2002 } \\ & \left(\mathrm{kg} \mathrm{ha}^{-1}\right)\end{aligned} \quad \Delta \mathrm{BT}\left(\right.$ ton ha $\left.^{-1} \mathrm{a}^{-1}\right)$

Brazo Riíto

$\begin{array}{ccccccc}\mathrm{Lr} & 679.4 & 11.4 & 37179.8 & 12.2 & 44261.8 & 3.5 \\ \mathrm{Ag} & 607.4 & 10.0 & 25621.5 & 11.1 & 32695.8 & 3.5 \\ \mathrm{Rm} & 66.2 & 14.1 & 8181.1 & 14.4 & 8740.9 & 0.3 \\ \mathrm{Ce} & 42.7 & 12.5 & & & & \\ \mathrm{Rb} & 7.4 & 2.8 & & & & \\ \mathrm{Pj} & 13.2 & 8.6 & & & & \\ \mathrm{Co} & 32.4 & 4.8 & & & & \\ \text { Total } & 1448.5 & 10.8 & 70982.3 & & 7.4\end{array}$

Valle de Los Cangrejos

$\begin{array}{cccc}\mathrm{Ag} & 610.7 & 8.8 & 19355.3 \\ \mathrm{Lr} & 895.2 & 4.5 & 5050.7 \\ \mathrm{Rm} & 48.8 & 9.8 & 2371.0 \\ \mathrm{Ce} & 2.4 & 0.0 & \\ \text { Total } & 1557.1 & 6.7 & 26777.0\end{array}$

Promedio para el delta

$\begin{array}{cccc}\mathrm{Ag} & 609.2 & 9.3 & 22131.6 \\ \mathrm{Lr} & 798.7 & 7.8 & 17713.3 \\ \mathrm{Rm} & 56.6 & 12.2 & 4859.3 \\ \mathrm{Ce} & 20.4 & 12.1 & \\ \mathrm{Rb} & 3.3 & 2.8 & \\ \mathrm{Pj} & 5.9 & 8.6 & \\ \mathrm{Co} & 14.5 & 4.8 & \\ \text { Total } & 1508.6 & 8.7 & 44704.2\end{array}$

Dq: diámetro promedio cuadrático; BT: biomasa total. Incrementos diamétricos sólo para RT. La biomasa total para 2002 no se asume como biomasa en pie ya que no considera pérdidas por mortalidad.

Dq: quadratic diameter; BT: total biomass. Diametric increments only for RT. Total biomass for 2002 is not standing living biomass, as mortality losses were not considered. 
de algunas especies a ciertos niveles de inundación hallada por Elster (1998). La presión por pastoreo y sequía prolongada afectan indudablemente la regeneración natural en el delta del río Ranchería y pueden generar grupos coetáneos discretos dentro del bosque. Las plántulas de $R$. mangle son las únicas que perduran en el ecosistema, pero el curso a estados de crecimiento superiores requiere investigación adicional. Es necesario también examinar más profundamente las consecuencias del pastoreo sobre el manglar para diseñar medidas de manejo eficaces en el delta del Ranchería.

La caída de hojarasca del delta del río Ranchería, (10.2-16.1 $\mathrm{mgha}^{-1} \mathrm{a}^{-1}, \alpha=0.05$; Fig. 5) es comparable con el ámbito superior global encontrado en la revisión de Saenger y Snedaker (1993) y otras publicaciones (Twilley et al. 1986, Bunt 1995, Twilley et al. 1997, Wafar et al. 1997, Barreiro-Güemes 1999, Betoulle et al. 2001, Ross et al. 2001, Sherman et al. 2003). Los valores hallados en el delta del río Ranchería son similares a los informados para manglares del Caribe que se están recuperando de eventos catastróficos de diferente índole (Zamorano 1983, Ross et al. 2001, Sherman et al. 2003). Aunque el dato del Ranchería parece contradecir la intuición en un bosque de bajo porte (sensu Vásquez 2000) y sometido a intensa aridez, monitoreos continuos de la caída de hojarasca en el delta del río Ranchería podrían confirmar que estos valores pueden ser la respuesta a los tensores fisicoquímicos o climáticos (Smith 1992). No es posible, sin embargo, descartar algún efecto del muestreo aplicado aquí.

La tasa de renovación foliar de 169 días calculada para el sitio de estudio se encuentra en el mismo ámbito encontrado por BarreiroGüemes (1999) en México (129-397 días) y por Ross et al. (2001) en Florida (189-281 días). De ello se deriva que dichas tasas son comparables, con una prolongación relacionada posiblemente con el aumento en latitud (Duke 1992).

Sánchez-Páez et al. (1998) registraron los incrementos de hasta $4.6 \%$ para $\mathrm{A}$. germinans en el Caribe colombiano, en un bosque monoespecífico maduro de tipo cuenca y estructura semejante al de RT, pero con mayor densidad. Los incrementos de $R$. mangle acá presentados (Cuadro 4) se asemejan a los encontrados por los mismos investigadores en bosques de franja en Islas del Rosario, aunque los de $L$. racemosa en RT fueron inferiores, lo que podría apuntar a respuestas específicas diferenciales. Jiménez (1990) hizo una revisión de trabajos que registran ámbitos de crecimiento diamétrico en manglar, entre 0.00 y $0.86 \mathrm{~cm} \mathrm{a}^{-}$ ${ }^{1}$, mismo ámbito de los datos aquí presentados, y registró un valor de $0.33 \mathrm{~cm} \mathrm{a}^{-1}$ en un bosque de $A$. bicolor en Costa Rica, estructuralmente semejante al del VC. Los crecimientos diamétricos que encontramos son entonces mayores a los esperados para manglares de clima seco, sometidos a largos períodos de aridez. El género Avicennia tolera mejor dichas condiciones y se expresa con abundancia y dominancia y, como se ve en este caso, en crecimiento diamétrico, incluso mayor al encontrado en sitios con condiciones ambientales más benignas.

La producción, que en RT valor duplica a $\mathrm{VC}$, no parece responder a diferencias en precipitación o evapotranspiración $\mathrm{y}$, a falta de mayor información, cabría reconocer la posibilidad de que los aportes de las alcantarillas que drenan allí aguas servidas favorezcan esta situación. Los valores de biomasa del Ranchería (Cuadro 5) se comparan con registros de Ross et al. (2001) en un bosque enano en Florida (22.3 $\left.\mathrm{mgha}^{-1}\right)$, donde la densidad es de 608 ind ha ${ }^{-1}$. Valores de este orden son documentados también por Saenger y Snedaker (1993) en bosques bajos en Australia, lo que apunta nuevamente a que ciertas características estructurales podrían responder de manera comparable a factores climáticos, con independencia de su composición específica.

La biomasa aérea en el Ranchería se contrasta con los bosques de franja y achaparrados mencionados por Saenger y Snedaker (1993), Chen y Twilley (1999), Ross et al. (2001), respecto de otros ribereños. Cabe especular, nuevamente, que esto se atribuye a la dramática estacionalidad de los aportes de agua, las variaciones en salinidad y la elevada insolación. 
El aporte anual de biomasa del bosque de manglar de Riohacha al ecosistema se halla en el mismo orden de magnitud señalado por Chen y Twilley (1999) para un sistema estuarino en Florida y su productividad se compara con Ross et al. (2001), quienes relacionaron valores para bosques en recuperación tras el paso de un huracán en el mismo estado.

Este estudio sugiere que manglares con baja biomasa en pie y sometidos a condiciones ambientales y antrópicas extremas pueden responder a éstas con una alta productividad, semejante a otros bosques en condiciones que podrían entenderse como más favorables para la producción de masa vegetal.

\section{AGRADECIMIENTOS}

Este estudio se desarrolló en el marco del proyecto cofinanciado por Colciencias "Estructura, fenología y demografía del manglar del Riíto y el Valle de los Cangrejos, delta del río Ranchería", código 1145-09-648-98. Los autores agradecen al personal y estudiantes vinculados al Centro de Investigaciones Científicas de la U. de la Guajira, a Jorge Vásquez y a Alexander Correa por suministrar información técnica. Agradecemos también a María Fernanda Buitrago por su contribución al contenido de este artículo.

\section{RESUMEN}

Se estudió de la supervivencia de plántulas y aumento de biomasa de Rhizophora mangle L., Avicennia germinans L. y Laguncularia racemosa (L.) Gaertn. f., principales especies de manglar del delta del río Ranchería, Colombia. Se encontró supervivencia de plántulas sólo de $R$. mangle. Se cuantificó también la tasa máxima de caída de hojarasca en el bosque; este valor se interpoló dentro de una regresión exponencial elaborada a partir valores máximos y anuales publicados en diferentes fuentes, y se halló un valor anual de $12.9 \mathrm{mgha}^{-1} \mathrm{a}^{-1}$. A partir ecuaciones alométricas elaboradas para la zona y otras de fuentes secundarias puede calcularse un aumento de biomasa en $7.4 \mathrm{mgha}^{-1} \mathrm{a}^{-1}$. La productividad del manglar del río Ranchería (20.2 mgha${ }^{1} \mathrm{a}^{-1}$ ) está representada en su mayoría por caída de hojarasca. Consideramos que esta productividad es alta para un bosque sometido a condiciones naturales y antrópicas tan desfavorables, como son la extrema estacionalidad y el continuo forrajeo de ganado ovino y caprino. Suponemos que la alta productividad se trata precisamente de una respuesta de la vegetación a la tensión ambiental.

Palabras clave: manglar, caída de hojarasca, crecimiento, supervivencia, productividad.

\section{REFERENCIAS}

Alongi, D., G. Wattayakorn, J. Pfitzner, F. Tirendi, I. Zagorskis, G. Brunskill, A. Davidson \& B. Clough. 2001. Organic carbon accumulation and metabolic pathways in sediments of mangrove forests in southern Thailand. Mar. Ecol 179: 85-103.

Anónimo. 1994a. In Informe final del Proyecto Manejo silvicultural del bosque de mangle del antiguo delta del río Sinú; estudio de regeneración natural del bosque de mangle. Forestales FVE Ltda. Corporación Autónoma Regional de los Valles del Río Sinú y San Jorge, Medellín, Colombia. $24 \mathrm{p}$.

Anónimo. 1994b. Calendario meteorológico 1994. Instituto colombiano de hidrología, meteorología y adecuación de tierras, HIMAT, Santa Fe de Bogotá, Colombia. $169 \mathrm{p}$.

Barreiro-Güemes, M.T. 1999. Aporte de hojarasca y renovación foliar del manglar en un sistema estuarino del sureste de México. Rev. Biol. Trop. 47: 729-737.

Barros, O., C. Pinzón \& L. Bastidas. 2002. Hidrología y dinámica del delta del río Ranchería y posibles efectos sobre el manglar del Riíto y Valle de los Cangrejos, Caribe colombiano. Trabajo de pregrado, Universidad de La Guajira, Riohacha, Colombia.

Betoulle, J.L., F. Fromard, A. Fabre \& H. Puig. 2001. Caractérisation des chutes de litière et des apports au sol en nutriments dans une mangrove de Guyane française. Can. J. Bot. 79: 238-249.

Bunt, J.S. 1995. Continental scale patterns in mangrove ecosystems. Hydrobiologia 295: 135-140.

Correa, M.A. 2002. Ecuaciones de biomasa y existencias de carbono de $A$. germinans y L. racemosa en el delta del Río Ranchería. Manuscrito Proyecto ColcienciasUniversidad de La Guajira, Medellín, Colombia. $11 \mathrm{p}$.

Cuignon, R. 1987. Estudio de la plataforma del Caribe colombiano, fase Guajira. Boletín Científico, Centro de Investigaciones Oceanográficas e Hidrográficas 7: 53-72. 
Chen, R. \& R.R. Twilley. 1999. Patterns of mangrove forest structure and soil nutrient dynamics along the Shark River estuary, Florida. Estuaries 22: 955-970.

Day, J.W., Jr., C. Coronado-Molina, F.R. Vera-Herrera, R. Twilley, V.H. Rivera-Monroy, H. Alvarez-Guillen, R. Day \& W. Conner. 1996. A 7 year above-ground net primary production in a southeastern Mexican mangrove forest. Aquat. Bot. 55: 39-60.

Díaz-Fuenmayor, K. 2001. Regeneración natural del manglar en el Rí́to y el Valle de los Cangrejos, delta del río Ranchería. Trabajo de pregrado, Universidad de La Guajira, Riohacha, Colombia. 65 p.

Duke, N.C. 1992. Mangrove floristics and biogeography, p 63-100. In A.I. Robertson \& D.M Alongi (eds.). Tropical mangrove ecosystems. Coast. Estuar. Stud. 41. Amer. Geophys. Union, Washington, D.C., EEUU.

Elster, C. 1998. Posibilidades de regeneración del manglar en la zona de la Ciénaga Grande de Santa Marta (Colombia). Deutsche Gesellshaft für Technische Zusammenarbeit (GTZ) Gmbh. Eschborn, Alemania. 68 p.

Ellison A.M. \& E.J. Farnsworth. 1993. Seedling, survivorship, growth and response to disturbance in Belizean mangal. Amer. J. Bot. 80: 1137-1145.

Fromard, F., H. Puig, E. Mougin, G. Marty, J.L. Betoulle \& L. Cadamuro. 1998. Structure, above-ground biomass and dynamics of mangrove ecosystems: new data from French Guiana. Oecologia 115: 39-53.

Gattuso, J.P., M. Frankignoulle \& R. Wollast. 1998. Carbon and carbonate metabolism in coastal aquatic ecosystems. An. Rev. Ecol. Syst. 29: 405-434

Holdridge, L.R. 1978. Ecología basada en las zonas de vida. Instituto Interamericano de Ciencias Agrícolas, San José, Costa Rica. 77 p.

Jiménez, J.A. 1990. The structure and function of dry weather mangroves on the Pacific coast of Central America, with emphasis on Avicennia bicolor forest. Estuaries 13: 182-192.

Jin-Eong, O., G.W. Khoon \& B.F. Clough. 1995. Structure and productivity of a 20-year-old stand of Rhizophora apiculata B1. mangrove forest. J. Biogeog. 22: 417 242.

Lacerda, L.D., J.E. Conde, B. Kjerfve, R. Álvarez-León, C. Alarcón \& J. Polanía. 2001. American Mangroves, p. 1-62. In L.D. Lacerda (ed.). Mangrove ecosystem, function and management. Springer, Berlín, Alemania.

Lema, L.F., J.L. Vásquez \& J. Polanía. 2002. Evaluación de criterios ecológicos para el manejo sostenible de los bosques de manglar en una zona semiárida del Caribe colombiano. Trabajo presentado en el II Congreso Forestal Latinoamericano, Ciudad de Guatemala, Guatemala.

Lugo A.E. \& S.C. Snedaker. 1974. The ecology of mangroves. An. Rev. Ecol. Syst. 5: 39-64.

Machiwa, J.F. \& R.O. Hallberg. 2002. An empirical model of the fate of organic carbon in a mangrove forest partly affected by anthropogenic activity. Ecol. Model.147: 69-83.

Martínez-Ramos, L. \& M. Ruiz-Ochoa. 2001. Caracterización de algunas propiedades físicas y físico-químicas del suelo manglárico en el Valle de los Cangrejos. Trabajo de pregrado, Universidad de la Guajira, Riohacha, Colombia. 70 p.

Maza, E. \& Y. Zárate. 2001. Hidroclimatología en el delta del río Ranchería: posibles efectos sobre la estructura del manglar en el Riíto y Valle de los Cangrejos, Caribe colombiano. Trabajo de pregrado, Universidad de La Guajira, Riohacha, Colombia. 124 p.

McKee, K.L. 1995. Seedling recruitment in a Belizean mangrove forest: effects of establishment ability and physico-chemical factors. Oecologia 101: 448-460.

Mehlig, U. 2001. Aspects of tree primary production in an equatorial mangrove forest in Brazil. Tesis de doctorado, Universidad de Bremen, Bremen, Alemania. 155p.

Rabinowitz, D. 1978a. Early growth of mangrove seedlings in Panamá, and an hypothesis concerning the relationship of dispersal and zonation. J. Biogeog. 5: 113-133.

Rabinowitz, D. 1978b. Mortality and initial propagule size in mangrove seedlings in Panamá. J. Ecol. 66: 45-51.

Ross, M.S., P. Ruiz, G.J. Telesnicki \& J.F. Meeder. 2001. Estimating above-ground biomass and production in mangrove communities of Biscayne National Park, Florida (USA). Wetl. Ecol. Manag. 9: 27-37.

Saenger, P. \& S.C. Snedaker. 1993. Pantropical trends in mangrove above-ground biomass and annual litterfall. Oecologia 96: 293-299. 
Sánchez-Páez, H., G.A. Ulloa-Delgado \& R. ÁlvarezLeón. 1998. Conservación y uso sostenible de los manglares del Caribe colombiano. Ministerio del Medio Ambiente/Asociación colombiana de reforestadotes-ACOFORE/Organización Internacional de Maderas Tropicales OIMT, Colombia. 224 p.

Sherman, R.E., T.J. Fahey \& P. Martinez. 2003. Spatial patterns of biomass and aboveground net primary productivity in a mangrove ecosystem in the Dominican Republic. Ecosystems 6: 384-398

Smith III, T.J. 1992. Forest structure, p 101-136. In A.I. Robertson \& D.M. Alongi (eds.). Tropical mangrove ecosystems. Coast. Estuar. Stud. 41. Amer. Geophys. Union, Washington D.C., EEUU.

Tanouchi, H., S. Nakamura, Y. Ochiai \& H. Azman. 2000. Profiles of species composition and aboveground biomass in a mangrove forest, Peninsular Malaysia. Japan Agricultural Research Quarterly 34: 147-151.
Twilley, R.R., A.E. Lugo \& C. Patterson-Zucca. 1986. Litter production and turnover in basin mangrove forests in southwest Florida. Ecology 67: 670-683.

Twilley, R.R., M. Pozo, V.H. García, V.H. Rivera-Monroy, R. Zambrano \& A. Bodero. 1997. Litter dynamics in riverine mangrove forests in the Guayas River estuary, Ecuador. Oecologia 111: 109-122.

Vásquez, J.L. 2000. Estructura de los bosques de mangle del Valle de los Cangrejos y el Riíto, delta del río Ranchería, Caribe Colombiano. Trabajo de pregrado, Universidad Nacional de Colombia Sede Medellín, Medellín, Colombia. 109 p.

Wafar, S., A.G. Untawale \& M. Wafar. 1997. Litter fall and energy flux in a mangrove ecosystem. Estuar. Coast. Shelf S. 44: 111-124

Zamorano, A.D. 1983. Productividad del manglar y su importancia para el pelagial de la Ciénaga Grande de Santa Marta. Tesis de Maestría, Universidad Nacional de Colombia, Santa Fe de Bogotá, Colombia. 122 p. 
